\title{
STELLAR PROMINENCES
}

\author{
ANDREW COLLIER CAMERON \\ School of Physics and Astronomy, University of St Andrews \\ North Haugh, St Andrews, SCOTLAND KY16 9SS.
}

\section{Introduction}

Stellar coronae, like the interstellar medium, are multi-phase media. Physical conditions in different parts of the corona allow material to exist in thermal equilibrium either as a tenuous X-ray plasma at temperatures of several million degrees, or in a denser form at temperatures low enough for hydrogen to recombine.

On the Sun, the various manifestations of the latter variety of coronal material are collectively known as prominences. This catch-all term is applied to a wide variety of solar coronal phenomena, ranging from the long-lived, quiescent prominences, to the more dynamic active-region prominences, cool post-flare loops, sprays and surges. All, however, owe their existence to the ability of the coronal magnetic field to provide both insulation against cross-field conduction, and mechanical support.

In the last decade, time-resolved spectroscopic studies of several classes of magnetically active cool stars have produced evidence that cool condensations can exist above the surfaces of other stars too. In deference to the solar analogy, these phenomena have also been labelled as stellar prominences, and in keeping with solar tradition they appear to represent a wide range of physical phenomena, not all of which have obvious counterparts on the Sun.

I shall begin this review by cataloguing briefly some of these phenomena, describing briefly the spectral signatures that led to their recognition. For the remainder of the talk, I shall concentrate on the potential uses of one particular subclass - the ones I like to call "slingshot prominences" - as probes of magnetic topology in the coronae of rapidly rotating young mainsequence stars. 


\section{Stellar prominences: observational evidence}

The earliest references to observations of prominence-like phenomena both appeared in 1983. Schröder (1983) found evidence of a prominence-like condensation above the surface of the $\mathrm{K}$ supergiant component of the eclipsing binary system 32 Cygni, in archival IUE observations using the B-star secondary as a pencil-beam probe of the K star's outer atmosphere. Shortly after the B star emerged from eclipse, a secondary dip was observed in the $1340 \AA, \lambda<1360 \AA$ UV continuum, which Schröder attributed to Rayleigh scattering at the HI ground state, and which yielded a column density $n_{\mathrm{H}} \simeq 10^{24} \mathrm{~cm}^{-2}$.

In the same year, Haisch et al. (1983) published simultaneous IUE and Einstein observations of a flare on Proxima Centauri. In the four minutes immediately following the peak of the X-ray flare, the lowest-energy channels in the the X-ray spectra from the Einstein Imaging Proportional Counter (IPC) showed a temporary decrease in flux. Haisch et al. interpreted this decrease to a temporary increase in the absorbing column overlying the flare site, from $n_{\mathrm{H}} \simeq 10^{18-19}$ to $n_{\mathrm{H}} \simeq 10^{20} \mathrm{~cm}^{-2}$. They pointed out that the absorption event could have been caused by the eruption of a prominence associated with the flare site, in the stellar analogue of a solar two-ribbon flare.

\subsection{STELLAR ANALOGUES OF ERUPTIVE PROMINENCES}

Subsequent multi-wavelength studies of active stars have continued to produce evidence of eruptive prominence activity associated with stellar flares.

Doyle et al. (1989) used IUE to obtain a set of MgII $h \& k$ spectra during a flare on the RS CVn binary II Peg. The MgII profiles showed two strong, narrow absorption features, one due to interstellar absorption, and the other present only in the flare spectrum and blue-shifted by $80 \mathrm{~km} \mathrm{~s}^{-1}$ relative to the star. This latter component appeared to have arisen in a cooler layer overlying the flare site. Byrne (1987) reported a transient red-shifted absorption feature in the $\mathrm{H} \alpha$ profile of this same star during late 1986, at a time when the amplitude of the star's light curve was exceptionally high. The $\mathrm{H} \alpha$ absorption was seen in a spectrum taken near the phase of maximum starspot visibility, and was interpreted as possible evidence for a large prominence-like structure with downflow velocities of up to $50 \mathrm{~km} \mathrm{~s}^{-1}$, overlying the spotted region. It is not clear whether this feature was in any way flare-related, but but its high downflow velocity suggests a dynamic phenomenon, possibly similar to solar post-flare loops.

Houdebine et al. (1993) calculated in some detail the kinetic energy and momentum budgets of a large flare on the dMe star AD Leo, based on multiwavelength observations of Doppler shifts and line asymmetries observed 
during the flare. They interpreted the data in terms of an erupting filament with a mass of order $6.4 \times 10^{13} \mathrm{~kg}$, followed by a slow coronal mass ejection. Significantly, they found that the kinetic energy associated with the mass ejections dominated the overall energy released in all forms.

\subsection{STELLAR ANALOGUES OF QUIESCENT PROMINENCES}

For the purposes of this discussion, I have opted to label as "quiescent" any observation of prominence-like activity that is not directly associated with a particular flare event, and which appears to persist for at least a day or so as a recognizable entity. As time is limited, I have chosen to concentrate on quiescent prominences for the remainder of this review, as I believe that these systems may have somewhat more to tell us about the topic of this conference, i.e. stellar surface structure.

In this category, a pencil-beam study of the eclipsing K0V + white dwarf binary V471 Tau by Jensen et al. (1986), using the EXOSAT spacecraft, revealed dips in the soft X-ray emission from the white dwarf. The dips were seen to repeat on successive 12-h orbital cycles of the binary before and after the eclipse of the white dwarf, indicating that the absorbing structures were fixed in the co-rotating frame of the binary system. At about the same time, Guinan et al. (1987) found transient absorption features in the UV lines of CII, CiII, Civ, SiII, SirII and Sirv, in IUE observations made just before and after the eclipse of the white dwarf. This indicated that the clumps contained material at a variety of temperatures, ranging from $10^{4}$ to $10^{5}$ $\mathrm{K}$, and were in some cases located as far as one stellar radius above the projected limb of the K0 dwarf.

\section{Optical signatures of quiescent stellar prominences}

Since then, spectral signatures of prominence activity have also been found at optical wavelengths. The strongest of these is the $\mathrm{H} \alpha$ line, which is also the most easily-observed optical signature in solar prominences. The most dramatic examples of optical absorption due to prominence activity are seen among rapidly rotating young main-sequence stars in the field and in young clusters such as the the $\alpha$ Persei and Pleiades clusters (ages 50 and 70 Myr respectively). The best-observed example of this class is AB Doradus, the star in which the prominence absorption was first found by Robinson \& Collier Cameron (1986). They observed transient $\mathrm{H} \alpha$ absorption features, which drifted at a steady rate from the blue to the red edge of the stellar rotation profile, over the course of an hour or so (fig. 1).

Subsequently, more detailed studies of the same object (Collier Cameron \& Robinson 1989a; Collier Cameron \& Robinson 1989b) revealed a complex system of circumstellar clouds, distributed between 3 and 9 stellar radii 
Rotation 10441

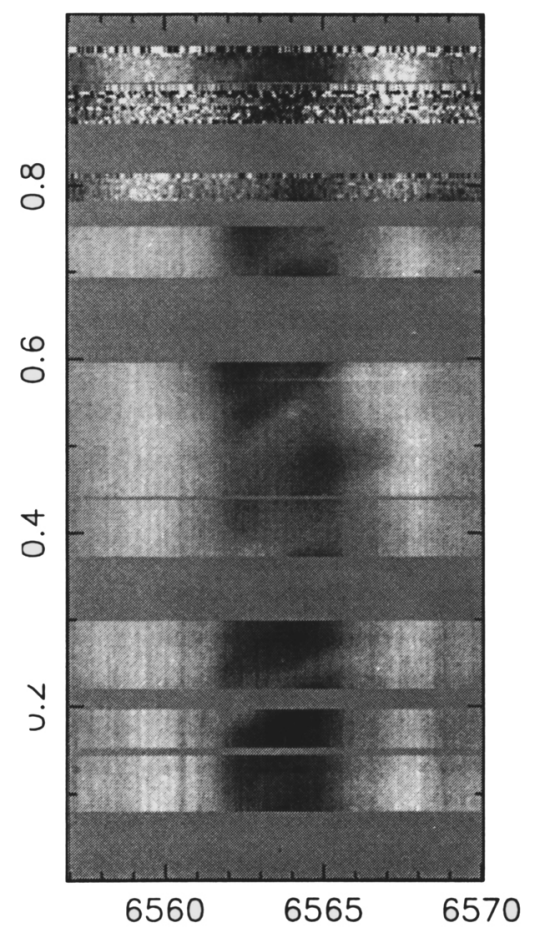

(a)
Rotation 10445

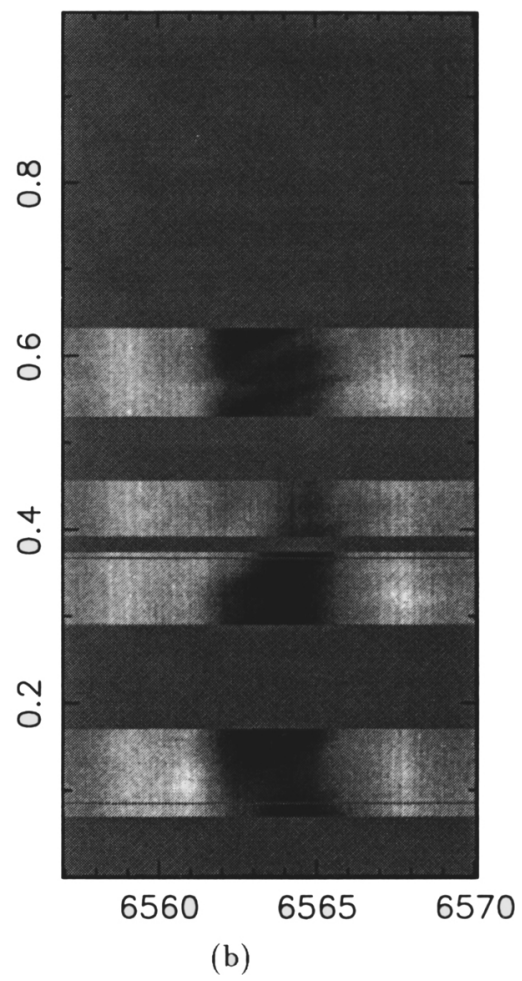

Figure 1. Phase-binned dynamic spectra of the $\mathrm{H} \alpha$ profile in AB Dor observed at CTIO and the AAT on the nights of 1995 November 15 and $17 \mathrm{UT}$. The rotation numbers and phases are derived from the ephemeris $\mathrm{HJD}=2444296.575+0.51479 E$. The greyscale runs from black at 0.80 times the local continuum level, to white at 1.02 times continuum.

from the rotation axis of the system and locked in solid-body rotation with the star. This placed the clouds beyond the Keplerian co-rotation radius, which in $\mathrm{AB}$ Dor lies some 2.6 stellar radii from the rotation axis, and implied that they must be confined against centrifugal ejection in the summits of closed magnetic loop structures (fig. 2).

Similar phenomena have been observed in the $\mathrm{H} \alpha$ profiles of four rapidly rotating G dwarfs in the $\alpha$ Per cluster (Collier Cameron \& Woods 1992); in the rapidly rotating M dwarf HK Aqr (Doyle \& Collier Cameron 1990); and in the rapidly rotating field star HD 197890, better known as Speedy Mic (Jeffries 1993). Indeed, the only well-observed rapid rotator in which the phenomenon has not been seen is BD +224409 , a low-mass member of the Local Association (Jeffries et al. 1994).

Hall \& Ramsey (1992) searched for anomalous $\mathrm{H} \alpha$ absorption features 


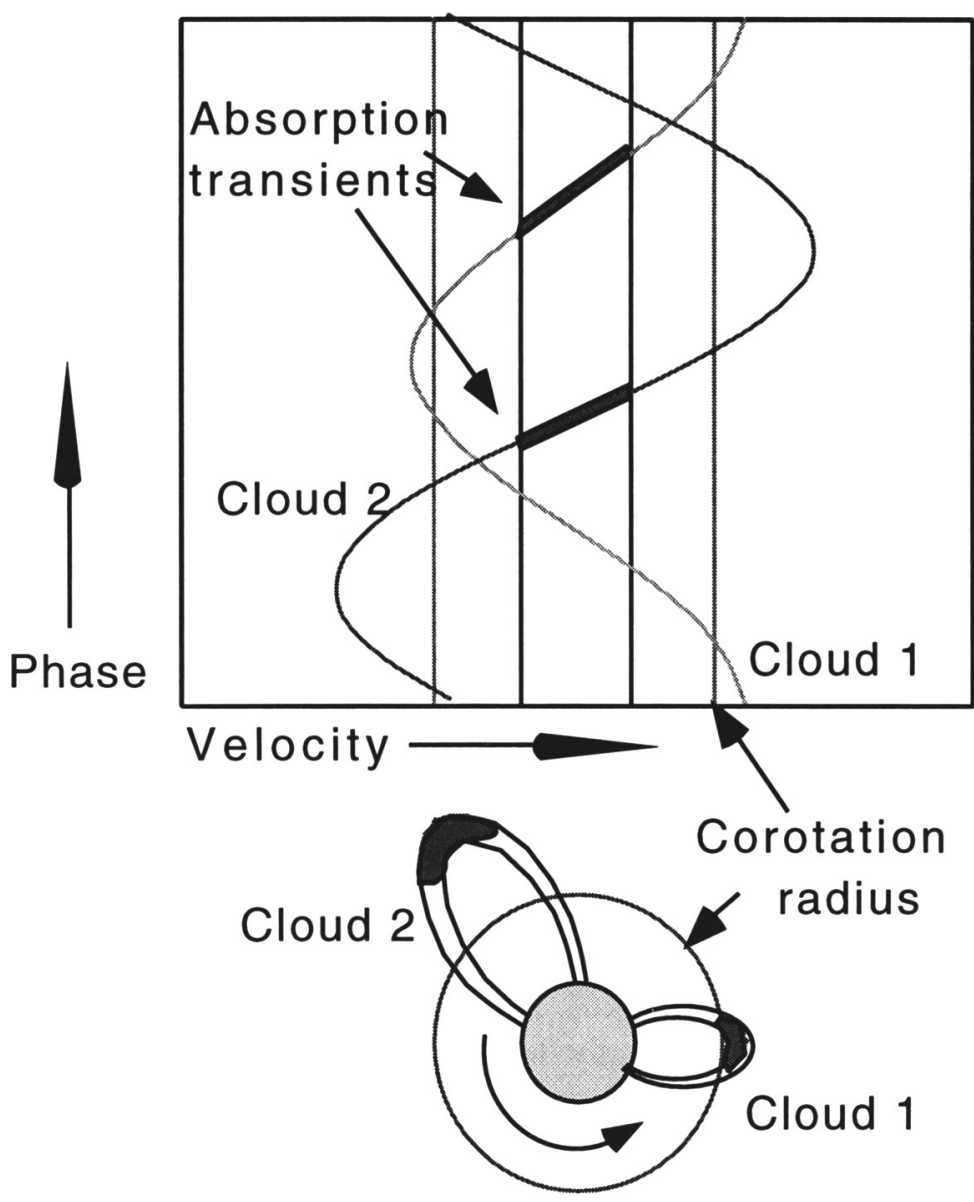

Figure 2. Schematic illustration of the relationship between the drift rate of an absorption transient and the distance of the cloud from the stellar rotation axis.

in a sample of ten eclipsing RS CVn systems. They found evidence of intrasystem absorbing material in eight of their targets. In a subsequent paper, Hall \& Ramsey (1994) were able to model the locations of the absorbing clouds and to infer that the structures appeared to be vertical, sheet-like structures with temperatures and electron densities similar to those of solar prominences, even though their physical extent was much greater than in the solar case. Since the evidence for prominence activity in binaries is discussed in greater detail in Jeffries' paper in this volume, however, I shall devote the remainder of this paper to single stars. 


\section{Slingshot prominence characteristics}

As is the case with solar prominences, the source function in the $\mathrm{H} \alpha$ line is dominated by scattering. Although the clouds are optically thick in $\mathrm{H} \alpha$, photons entering the cloud are unlikely to be destroyed by collisional deexcitation, and so are eventually scattered out of the cloud in random directions. The surface brightness of the clouds thus depends on the chromospheric $\mathrm{H} \alpha$ flux impinging on the cloud from beneath, and on the geometrical dilution factor. The latter is on the order of 0.01 for a cloud located near the co-rotation radius. When viewed in silhouette against the stellar disc in the core of the $\mathrm{H} \alpha$ line, the clouds thus appear almost completely dark. This allows us to estimate the area of a given cloud from the equivalent width $W$ of its absorption profile:

$$
W=\bar{w} a,
$$

where $\bar{w}$ is the area-weighted average EW of the cloud's absorption profiles along individual lines of sight, and $a$ is the fraction of the stellar disc obscured by the cloud. If the difference in rotational Doppler shift between the leading and trailing edges of the cloud is narrower than the saturated portion of the cloud's intrinsic $\mathrm{H} \alpha$ absorption profile, then the cloud area can be estimated very simply as $a=1-R_{0}$, where $R_{0}$ is the central residual intensity of the line profile.

Since the larger transients typically have $1-R_{0} \simeq 0.1$ to 0.25 , this implies that the linear dimensions of a typical cloud are a few tenths of the stellar diameter. Collier Cameron et al. (1990) used an extension of this technique to infer the column density of $\mathrm{Ca}$ II through several clouds by interleaving $\mathrm{Ca}$ II $H \& K$ and $\mathrm{H} \alpha$ spectra, and using the cloud area information to infer the intrinsic EW of the $\mathrm{Ca}$ II $H \& K$ absorption profiles along individual lines of sight. By assuming solar abundances, they inferred column densities $N_{\mathrm{H}} \simeq 10^{20} \mathrm{~cm}^{-2}$, and temperatures of order 8000 to 9000 $\mathrm{K}$. The total cloud area $\left(a \simeq 3 \times 10^{21} \mathrm{~cm}^{2}\right)$ then yields an overall mass of order 2 to $6 \times 10^{17} \mathrm{~g}$, which is two to three orders of magnitude greater than the total mass of a large solar quiescent prominence, $M \simeq 10^{15} \mathrm{~g}$.

It should be noted that the linear dimensions of the condensations are considerably greater than the pressure scale height for such cool material, which is on the order of a few hundred $\mathrm{km}$. For this reason it seems plausible that, like solar quiescent prominences, the slingshot prominences may have a highly filamented structure with a relatively low filling factor. In this case, we should expect to find material at transition-region temperatures associated with a thin sheath wrapped around each filament, at the interface with the hot ambient corona. This expectation has been vindicated in a recent simultaneous $H S T$ and ground-based campaign on AB Dor, in which 
absorption features were seen to cross the profiles of the $\mathrm{C}$ IV resonance lines at the same time as $\mathrm{H} \alpha$ transients observed at CTIO (see also Walter's paper in this volume). This also suggests that the clumps of material seen in absorption in the V471 Tau system by Guinan et al. (1987) may be similar in nature to the AB Dor prominences.

\section{Short-term evolution}

The number of prominence complexes present in the observable slice of the corona is typically 6 to 8 , although there can be wide variations in this number from year to year. As is evident from Fig. 1, significant changes can occur in the prominence system on timescales as short as one or two days.

In particular, new clouds have been observed to form on $A B$ Dor at rotation phases where no cloud was present two stellar rotations (approximately 25 hours) previously. Indeed, on average we tend to see one new cloud appear and/or one cloud disappear, between successive nights of observation. This, together with the average number of clouds seen on a given night, implies that the lifetime of a typical cloud is on the order of one week.

The formation timescale is consistent with the cooling time of coronal plasma at temperatures of order $10^{7} \mathrm{~K}$. This, combined with the prominences' apparent preference for a formation site just outside the co-rotation radius, suggests that the formation of slingshot prominences may be triggered by centrifugal compression of coronal plasma in loops whose summits extend beyond the co-rotation radius. Several authors, including Collier Cameron (1988), Jardine \& Collier Cameron (1991), and Ferreira \& Jardine $(1995 \mathrm{a}, \mathrm{b})$ have addressed the problem of the thermal and mechanical structure of coronal loops in which rapid rotation leads to a reversal of the gravitational potential gradient near the summit (see also van den Oord's paper in this volume). There appears to be no problem with the existence of appropriate loop solutions, nor in feeding the growth of a prominence with a siphon flow up the loop legs once thermal collapse has occurred at the summit, provided the field strengths near the loop footpoints are of order a kilogauss or more.

\section{Relationship to surface structures}

The observations described above imply the presence of large, closed magnetic loop structures that can confine and support large condensations of cool material well above the stellar surface. Since these magnetic structures must clearly be anchored in the stellar convective zone, their foot- 
points should be associated with recognizable magnetic structures at the photospheric level.

Several authors have searched for spatial correlations with starspots. Byrne (1987) noted that the red-shifted Ha absorption seen in II Peg in 1986 was seen against the star's most heavily spotted hemisphere, while Guinan et al. (1987) noted a tendency for the UV line-absorbing condensations to appear above the more heavily spotted limb of V471 Tau.

Given the difficulty of pinpointing starspot positions from photometry alone, however, it is hard to draw firm conclusions from these observations. In the case of $\mathrm{AB}$ Dor, however, simultaneous Doppler imaging and prominence-mapping over three observing seasons (1992 January to 1993 November) has shown the star to possess a complex starspot distribution with a coherence time for individual features of order one year (Collier Cameron 1995; Unruh et al. 1995). The prominence distribution has a much shorter coherence time, of order one week (Collier Cameron \& Robinson 1989b). To date, we have found no clear correlation between starspot locations and prominence formation sites. This is illustrated nicely by comparing Doppler images of AB Dor obtained in 1992 January (fig. 3 ) and December (fig. 4), with trailed $\mathrm{H} \alpha$ spectra phased such that each transient appears above the corresponding longitude in the accompanying Doppler image. While the starspot distribution is essentially unaltered - at low latitudes, at least - the prominence distribution has changed beyond recognition.

The AB Dor prominences are unlikely to be anchored at low latitudes. If the star's luminosity is similar to that of a Pleiades K0 dwarf, its distance should be between 16 and $19 \mathrm{pc}$, yielding an axial inclination of $60 \circ$ to the line of sight (from $v \sin i, P_{\text {rot }}$ and its Barnes-Evans angular diameter). This is also the inclination angle that gives the best maximum-entropy surface images. Any material located near the co-rotation radius that transits the stellar disc must therefore lie between 0.5 and 2 stellar radii above the stellar equatorial plane; for the few clouds that have been seen as far at 6 stellar radii from the rotation axis, the distances from the plane are correspondingly greater. For material to be supported this far above the plane suggests that (i) the confining structures may be located at relatively high latitudes on the stellar surface, and (ii) there must be a strong vertical magnetic pressure gradient, perhaps due to other, similar structures at lower latitudes which do not transit the disc and so are unobservable.

Observations of other stars such as the G dwarfs in the $\alpha$ Persei cluster confirm that prominence systems can be seen transiting the discs of stars with a range of axial inclinations. The only rapid rotator so far discovered not to show $\mathrm{H} \alpha$ absorption transients is $\mathrm{BD}+22^{\circ} 4409$, which according to Jeffries et al. (1994) appears to have a low axial inclination. If this result is 
1992 January 18: H alpha dynamic spectrum

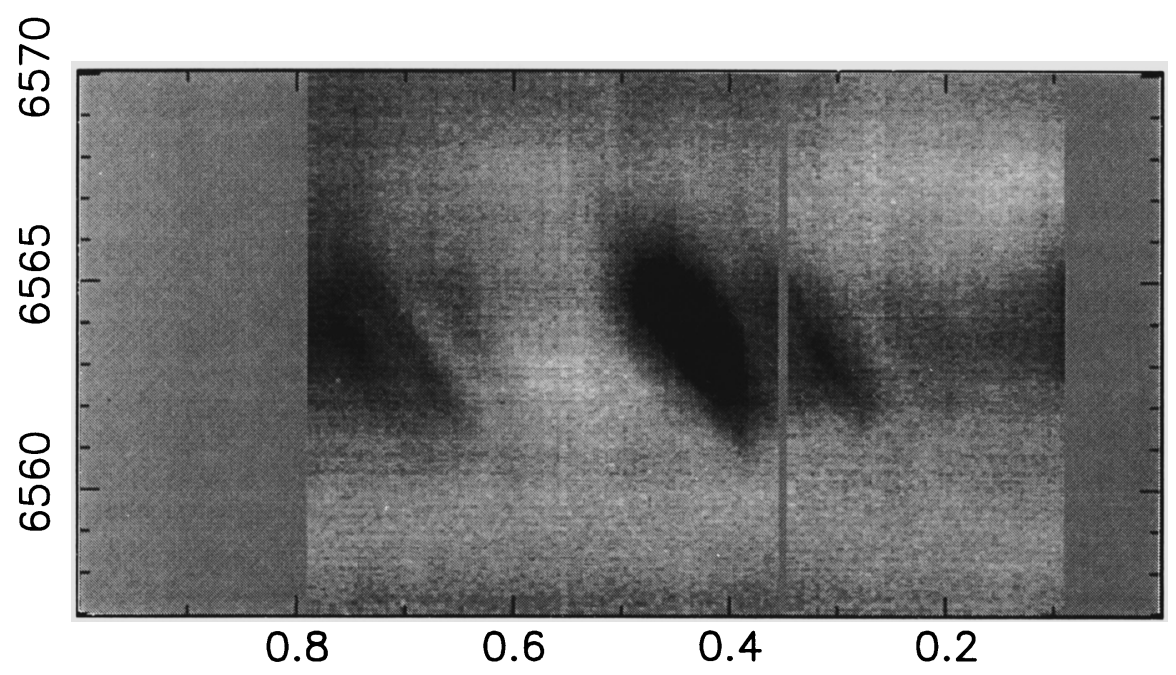

(a)

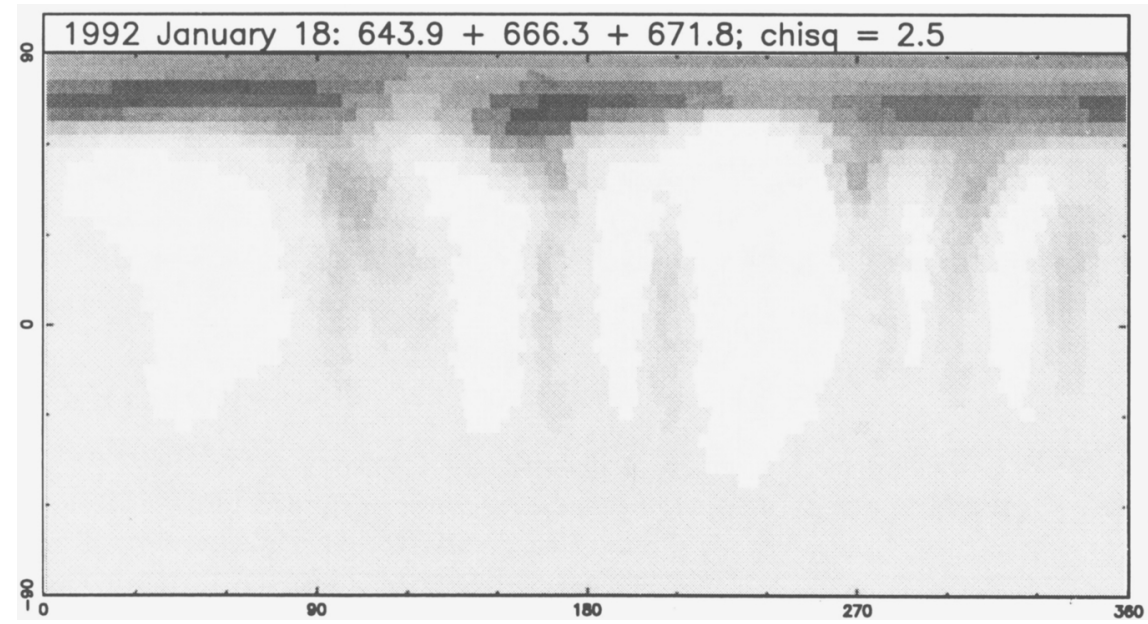

(b)

Figure 3. (a) $\mathrm{H} \alpha$ dynamic spectrum and (b) stellar surface image of AB Doradus, taken at the AAT on the night of 1992 January 18 UT. 
1992 December 14: H alpha dynamic spectrum

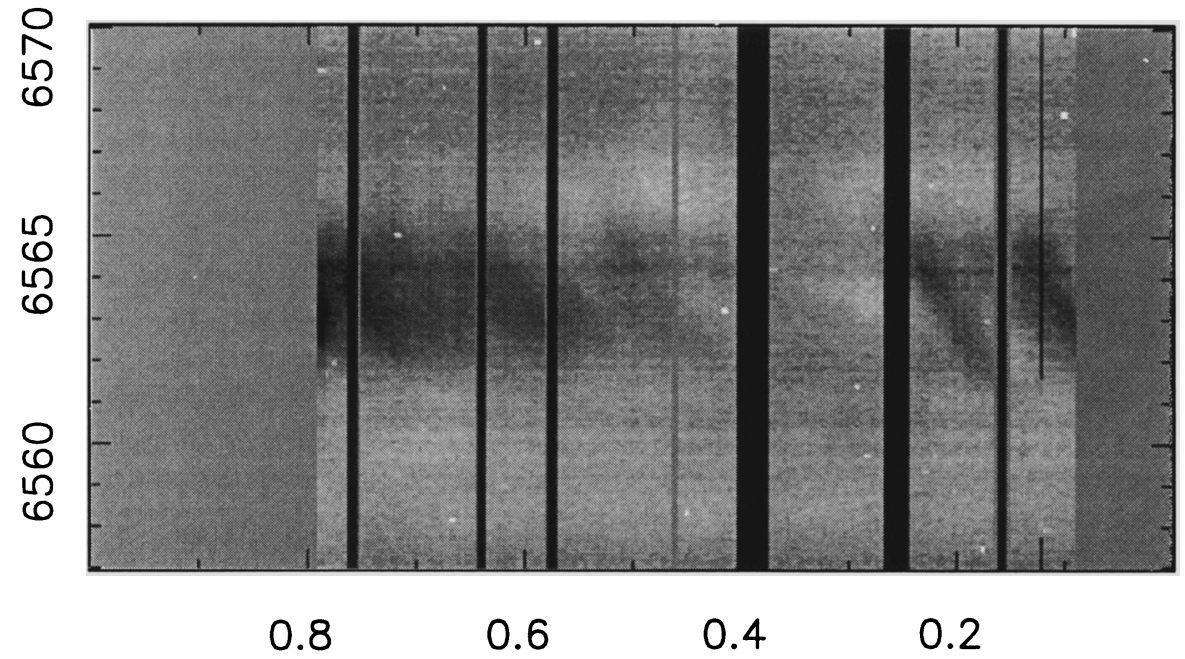

(a)

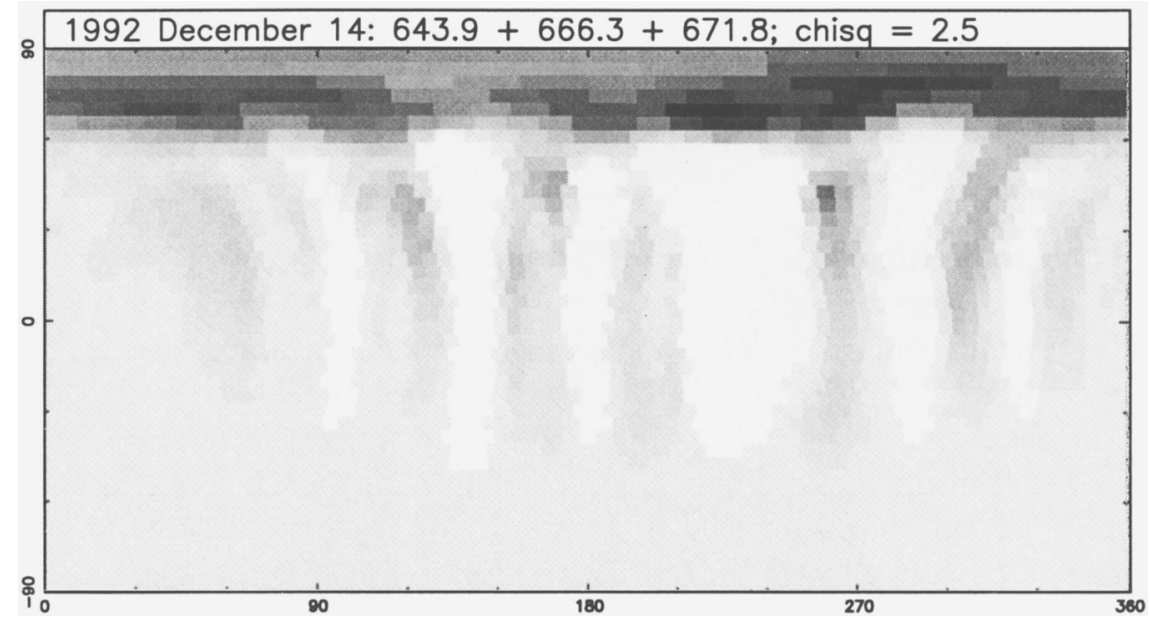

(b)

Figure 4. (a) $\mathrm{H} \alpha$ dynamic spectrum and (b) stellar surface image of AB Doradus, taken at the AAT on the night of 1992 December 14 UT. The phase coverage of these observations is similar to that of 1992 January 18 (Fig. 3). 
confirmed for other low-inclination stars, it would imply that the slingshot prominence systems in these stars are principally confined to a thick torus lying near or just outside the co-rotation radius.

The detailed relationship between slingshot prominences and the most obvious magnetic structures at the level of the photosphere - starspots remains elusive. The solar analogy, however, suggests that this may not be too surprising. On the Sun, quiescent prominences lie along magnetic polarity inversion lines separating large unipolar regions. In particular, the polar crown of prominences in each hemisphere straddles the boundary of the polar coronal hole, at considerably higher latitudes than the bulk of spot activity.

\section{Summary and unanswered questions}

There is now abundant observational evidence that active stars show scaledup versions of both quiescent and eruptive prominence activity. Among the best-studied examples of this type of structure are the slingshot prominence systems that are found near the co-rotation radii of many rapidly rotating, single stars. The locations of these condensations are a powerful probe for mapping the locations of extended closed loop structures in these stars' coronae. The mechanical and thermal structure of these loops, and the processes that lead to the formation of these condensations, remain a challenge for theorists. On the observational side, the discovery of material at transition-region temperatures opens the way to studying the lateral energy balance in filamentary sub-structures. The expectation that these closed loops should straddle magnetic polarity inversion lines between large-scale unipolar regions, with kilogauss fields at their footpoints, should be amenable to testing with advanced surface imaging techniques such as Zeeman Doppler imaging.

\section{REFERENCES}

Byrne P. B., 1987, in Linsky J. L., Stencel R. E., eds, Lecture Notes in Physics, Vol. 291: Cool Stars, Stellar Systems and the Sun. Springer-Verlag, p. 491

Collier Cameron A., 1988, MNRAS, 233, 235

Collier Cameron A., 1995, MNRAS, 275, 534

Collier Cameron A., Robinson R. D., 1989a, MNRAS, 236, 57

Collier Cameron A., Robinson R. D., 1989b, MNRAS, 238, 657

Collier Cameron A., Woods J. A., 1992, MNRAS, 258, 360

Collier Cameron A., Duncan D. K., Ehrenfreund P., Foing B. H., Kuntz K. D.,

Penston M. V., Robinson R. D., Soderblom D. R., 1990, MNRAS, 247, 415

Doyle J. G., Collier Cameron A., 1990, MNRAS, 244, 291

Doyle J. G., Byrne P. B., van den Oord G. H. J., 1989, A\&A, 223, 219

Ferreira J. M. T. S., Jardine M., 1995a, A\&A, 298, 172

Ferreira J. M. T. S., Jardine M., 1995b, A\&A, In press 
Guinan E. F., Wacker S. W., Baliunas S. L., Loeser J. G., Raymond J. C., 1987, in New Insights in Astrophysics: Eight Years of UV Astronomy with IUE. ESA SP-263, p. 197

Haisch B. M., Linsky J. L., Bornmann P. L., Stencel R. E., Antiochos S. A., Golub L., Vaiana G. S., 1983, ApJ, 267, 280

Hall J. C., Ramsey L., 1992, AJ, 104, 1942

Hall J. C., Ramsey L., 1994, AJ, 107, 1149

Houdebine E. R., Foing B. H., Doyle J. G., Rodono M., 1993, A\&A, 278, 109

Jardine M. M., Collier Cameron A., 1991, Solar Phys., 131, 269

Jeffries R. D., 1993, MNRAS, 262, 369

Jeffries R. D., Byrne P. B., Doyle J. G., Anders G. J., James D. J., Lanzafame A. C., 1994, MNRAS, 270, 153

Jensen K. A., Swank J. H., Petre R., Guinan E. F., Sion E. M., Shipman H. L., 1986, ApJ, 309, L27

Robinson R. D., Collier Cameron A., 1986, Proc. Astron. Soc. Aust., 6, 308

Schröder K. P., 1983, A\&A, 124, L16

Unruh Y. C., Collier Cameron A., Cutispoto G., 1995, MNRAS, In press 